\title{
EDITORIAL
}

\section{The Role of $\mathrm{Ca}^{2+}$-activated $\mathrm{K}^{+}$Channels of Intermediate Conductance in Glioblastoma Malignancy}

Glioblastoma is the most common and malignant brain tumor. In spite of the extensive basic and clinical studies carried out over many years in this research area, still one-third of patients die before one year from diagnosis, and less than 5\% survive at five years. A major obstacle with glioblastoma is its high migratory and invasive potential into the healthy brain tissue that prevents complete surgical removal of tumor cells and effective radiotherapic treatment. The outcome of the disease is further worsen by the presence in the tumor mass of subpopulations of highly tumorigenic, stem-like glioblastoma cells that possess even higher invasive power, chemo and radioresistance than normal tumor cells, and are responsible for the frequent tumor relapses which are the actual cause of the fatal outcome of the disease.

Glioblastoma is characterized by a large number and variety of genetic mutations that heavily disregulate major signaling pathways controlling cell survival, proliferation, and invasion: the allmarks of glioblastoma malignancy. Most relevant with the scope of this mini issue are those pathways controlling the expression of ion channels, the transmembrane proteins endowed with gating and permeation structures that allow the regulated passage of ions. Ion channels have been deeply involved in several cellular functions that determine glioblastoma malignancy. Their most relevant contribution in connection with glioblastoma progression consists in controlling two basic cellular parameters underlying glioblastoma aggressiveness, the cell volume and the intracellular $\mathrm{Ca}^{2+}$ changes $\left(\left[\mathrm{Ca}^{2+}\right]_{\mathrm{i}}\right)$.

Changes of cell volume are commonly attained by regulating $\mathrm{K}^{+}$and $\mathrm{Cl}^{-}$transmembrane fluxes, which are followed by the osmotically driven water. For example, a premitotic volume condensation (PVC) is required for glioblastoma cells to switch from a bipolar into a round cell morphology just prior to cell division. Likewise, a cell volume reduction - the apoptotic volume decrease (AVD) - is observed during the staurosporine-induced apoptosis of glioblastoma cells. Cell migration and invasion through the narrow extracellular spaces of brain parenchyma also require major changes in cell volume, again sustained by $\mathrm{K}^{+}$and $\mathrm{Cl}^{-}$transmembrane fluxes. As for the Ca ${ }^{2+}$ signals in the progression of glioblastoma, recent research shows to be critically dependent on ion channels. Besides sustaining directly the $\mathrm{Ca}^{2+}$ influxes (through voltage dependent and voltage independent $\mathrm{Ca}^{2+}$-permeable channels), they can influence the entry of extracellular $\mathrm{Ca}^{2+}$ by modulating the membrane potential that controls the driving force for $\mathrm{Ca}^{2+}$ influx. For instance, $\mathrm{Ca}^{2+}$ oscillations, instrumental to glioblastoma cell migration, can be significantly affected by the membrane hyperpolarization determined by the activity of $\mathrm{K}$ channels.

Perhaps the best suited ion channels to play a significant role in glioblastoma progression are the $\mathrm{Ca}^{2+}$-activated $\mathrm{K}(\mathrm{KCa})$ channels, as they are at the cell crossroad where $\mathrm{Ca}^{2+}$ influx, membrane potential and outward $\mathrm{K}^{+}$fluxes meet. At this intersection, $\mathrm{KCa}$ channels integrate these signals and as result modulate a large array of cellular processes including those relevant to glioblastoma malignancy.

$\mathrm{KCa}$ channels were originally classified according to their single-channel conductance into big (BKCa), intermediate (IKCa), and small-conductance ( $\mathrm{SKCa}) \mathrm{K}^{+}$channels. Later studies based on genetic relationship, voltage dependence and mechanisms of $\mathrm{Ca}^{2+}$ activation restricted this classification to two major subfamilies: one, exhibiting large unitary conductance and gated by the cooperative action of membrane depolarization and $\left[\mathrm{Ca}^{2+}\right]_{i}$, include $\mathrm{BKCa}$ as main representative, while the other comprise IKCa and SKCa, which display much smaller conductance and are gated solely by $\left[\mathrm{Ca}^{2+}\right]_{\mathrm{i}}$. Moreover, unlike $\mathrm{BKCa}$, the members of the second group do not bind $\mathrm{Ca}^{2+}$ directly, but rather detect $\mathrm{Ca}^{2+}$ via calmodulin, which is constitutively bound to their $\mathrm{C}$-terminal region. Binding of $\mathrm{Ca}^{2+}$ to calmodulin results in conformational changes that are in turn responsible for channel gating. KCa channels evolution analysis confirmed their grouping into two well defined and distantly related subfamilies.

This mini issue focuses on the IKCa channel, among the $\mathrm{KCa}$ channels arguably the one playing the most significant role in glioblastoma progression. The IKCa channel has been shown directly involved in both cell volume regulation and intracellular Ca ${ }^{2+}$ signaling, two primary parameters controlling cell proliferation, migration and apoptosis, hallmarks of glioblastoma malignancy.

The first contribution describes the main structural, biophysical and modulatory properties of the KCa3.1 channel, and provides a detailed account of experimental data on its expression in glioblastoma cells, as compared to normal brain tissue. The effects KCa3.1 channel activity on basic cellular functions in glioblastoma, such as cell volume regulation and intracellular calcium are also reviewed.

The second contribution briefly reviews the available compounds known to potently modulate KCa3.1 channel activity, their binding sites and mechanisms of action, and then discusses the potential use of these compounds for the treatment of brain tumors, also based on their brain penetration.

The third contribution summarizes our current knowledge on the molecular signaling upstream and downstream, and the effector functions of IK channel activity in tumors in general, and in particular in glioblastoma cells. New in vitro data are also presented on IK channel expression in subtypes of glioblastoma stem-like cells.

The fourth contribution reviews the currently available evidence for a functional role of KCa3.1 channels in in vivo glioblastoma tissue, with the object of establishing whether modulating KCa3.1 activity can be an adjuvant therapeutic approach to classic chemotherapy, to contrast tumor growth and prolong patient's survival. 